Review Article

\title{
Evidence Supporting the Hypothesis That Inflammation-Induced Vasospasm Is Involved in the Pathogenesis of Acquired Sensorineural Hearing Loss
}

\author{
Michael Eisenhut (D) \\ Luton and Dunstable University Hospital NHS Foundation Trust, Lewsey Road, Luton LU40DZ, UK \\ Correspondence should be addressed to Michael Eisenhut; michael_eisenhut@yahoo.com
}

Received 17 June 2019; Revised 4 October 2019; Accepted 19 October 2019; Published 6 November 2019

Academic Editor: Gerd J. Ridder

Copyright (c) 2019 Michael Eisenhut. This is an open access article distributed under the Creative Commons Attribution License, which permits unrestricted use, distribution, and reproduction in any medium, provided the original work is properly cited.

\begin{abstract}
Sensorineural hearing loss is mainly acquired and affects an estimated 1.3 billion humans worldwide. It is related to aging, noise, infection, ototoxic drugs, and genetic defects. It is essential to identify reversible and preventable causes to be able to reduce the burden of this disease. Inflammation is involved in most causes and leads to tissue injury through vasospasm-associated ischemia. Vasospasm is reversible. This review summarized evidence linking inflammation-induced vasospasm to several forms of acquired sensorineural hearing loss. The link between vasospasm and sensorineural hearing loss is directly evident in subarachnoid haemorrhage, which involves the release of vasoconstriction-inducing cytokines like interleukin-1, endothelin-1, and tumour necrosis factor. These proinflammatory cytokines can also be released in response to infection, autoimmune disease, and acute or chronically increased inflammation in the ageing organism as in presbyacusis or in noise-induced cochlear injury. Evidence of vasospasm and hearing loss has also been discovered in bacterial meningitis and brain injury. Resolution of inflammation-induced vasospasm has been associated with improvement of hearing in autoimmune diseases involving overproduction of interleukin-1 from inflammasomes. There is mainly indirect evidence for vasospasm-associated sensorineural hearing loss in most forms of systemic or injury- or infection-induced local vascular inflammation. This opens up avenues in prevention and treatment of vascular and systemic inflammation as well as vasospasm itself as a way to prevent and treat most forms of acquired sensorineural hearing loss. Future research needs to investigate interventions antagonising vasospasm and vasospasm-inducing proinflammatory cytokines and their production in randomised controlled trials of prevention and treatment of acquired sensorineural hearing loss. Prime candidates for interventions are hereby inflammasome inhibitors and vasospasm-reducing drugs like nitric oxide donors, rho-kinase inhibitors, and magnesium which have the potential to reduce sensorineural hearing loss in meningitis, exposure to noise, brain injury, arteriosclerosis, and advanced age-related and autoimmune disease-related inflammation.
\end{abstract}

\section{Introduction}

Vasospasm is a consistent feature of all forms of cerebral inflammation including forms caused by infections like bacterial meningitis, cerebral malaria, and sterile vascular inflammation as detectable in diabetic ketoacidosis and brain injury $[1,2]$. Vasospasm is hereby mediated by depletion of nitric oxide and direct effects of interleukin-1 (IL1), tumor necrosis factor (TNF), and endothelin-1. Cerebral vasospasm has been associated with cerebral ischemia and subsequent neurological deficits. The cochlear hair cells are supplied almost exclusively by a single terminal artery, which is the labyrinthine artery also called the spiral modiolar artery, a branch of the anterior inferior cerebellar artery (AICA), which terminates radially in the lateral cochlear wall, thereby forming the stria vascularis. Cochlear hair cells have high oxygen consumption and poor tolerance to hypoxia. In "vasospasm-related" sensorineural hearing loss (SNHL), which is caused by ischemia, the pattern should be a sudden loss of hearing across several frequencies, which in most cases would start or be exclusively unilateral because a synchronicity of vasospasm occurring at exactly the same time in both ears is unlikely unless caused by a factor which acts equally on both labyrinthine arteries like an ototoxic 
drug or equal exposure to a source of noise. Trauma, infection, autoimmune-, or arteriosclerosis-related transient vascular stenosis is likely due to an asymmetrical exposure of the supply artery to cytokines causing vasospasm. The hearing loss should be sensorineural but reversible in a percentage of patients indicating that not hair cell loss but temporary hair cell dysfunction secondary to temporary ischemia as found in a transient vasospasm is responsible in some cases. In this review, I have attempted to summarize the evidence for a link between inflammation-induced vasospasm and acquired SNHL as well as approaches to prevention and treatment.

\section{Methods}

2.1. Inclusion Criteria. Included were the following studies:

(a) Reports providing evidence relating to conditions associated with inflammation and SNHL and/or a focus on a potential association between inflammation-induced vasospasm and SNHL. As indicator of vasospasm was in the interpretation hereby taken that a proportion of people affected by a condition associated with SNHL showed laterality/ and or reversibility of the SNHL.

(b) Both animal and human studies.

(c) All studies reporting randomised controlled trials of anti-inflammatory or vasospasm-relieving drugs in prevention and/or treatment of SNHL.

(d) Studies which reported on the pathophysiology of cytokine-induced hearing loss or vasospasm.

2.2. Exclusion Criteria. Excluded were the following studies:

(a) Studies in which patients with conductive hearing loss were investigated.

(b) Studies not reported in English language.

(c) Case reports on effect of treatment modalities on SNHL.

(d) Nonrandomised studies with regard to treatment trials in humans.

(e) Studies on compromise of vascular supply by arterial wall thickening, e.g., in vasculitis and endoluminal stenosis like in subendothelial atherosclerotic proliferation or in obstruction of the vascular lumen by embolism.

2.3. Database Search. Searches using the following keywords were carried out in September 2019: (i) "hearing loss" and "vasospasm" in the PubMed database and (ii) "sensorineural hearing loss" in the Cochrane Library database.

References of relevant articles were searched and incorporated where indicated. For flow chart of literature search, see Figure 1.

2.4. Categorisation of Evidence of a Link between SNHL and Inflammation-Induced Vasospasm. In the descending order of strength of a potential causal association of inflammationinduced vasospasm and SNHL, conditions were categorized into 3 categories:

(1) "Direct evidence" for inflammation-associated vasospasm causing SNHL studies where there was evidence of vasospasm on in vivo dynamic imaging like Doppler flow studies in patients with conditions with SNHL more than in patients without the same conditions. Evidence regarding vasoconstrictors and treatment with ant-inflammatory agents and vasodilators was similarly labelled if based on a model with direct evidence.

(2) "Features consistent with inflammation-associated vasospasm-induced SNHL" and the associated treatment addressing and/or leading to a change in those features.

(3) "Conditions associated with increased risk of inflammation associated with vasospasm-induced SNHL." All forms of inflammation involving vasoconstriction causing cytokines were regarded as putting the cochlear perfusion at risk of vasospasminduced compromise.

\section{Results}

\subsection{Inflammatory and/or Vasospastic Aspects of Clinical Entities Associated with Hearing Loss}

3.1.1. Sudden Hearing Loss. Direct evidence for a vasospasm as etiological factor in acquired SNHL is the analysis of data from children with sudden SNHL where influence of noise exposure, medication and drugs including illicit substances, and exposure to chemicals at the work place which could cause temporary hair cell dysfunction is much less likely. A pooled analysis of 226 cases (ears) of sudden SNHL in children from two centres in China demonstrated $85 \%$ of children had unilateral hearing loss, of which the largest proportion was profound (51\%). The hearing loss was reversible to a variable extent (including any form of recovery) in $48.6 \%$ [3, 4], and there was no dominance of a side. Evidence has emerged that microvascular dysfunction is associated with sudden onset SNHL. Microvascular dysfunction was hereby measured using videocapillaroscopic examination of the nailfold, measuring the capillary density (CD) and postocclusive reactive hyperaemia (PORH) values. Patients with sudden onset SNHL had significantly lower $\mathrm{CD}$ and PORH values [5]. Surgical induction of vasospasm in the labyrinthine artery has been shown to cause reversible SNHL [6].

3.1.2. Autoimmune Diseases. The hearing loss found associated with autoimmune-induced inflammation has features consistent with inflammation-associated vasospasm-induced sensorineural hearing loss. The hearing loss in autoimmune-induced inflammation is potentially reversible, and anti-inflammatory treatment can prevent and treat SNHL [7]. 


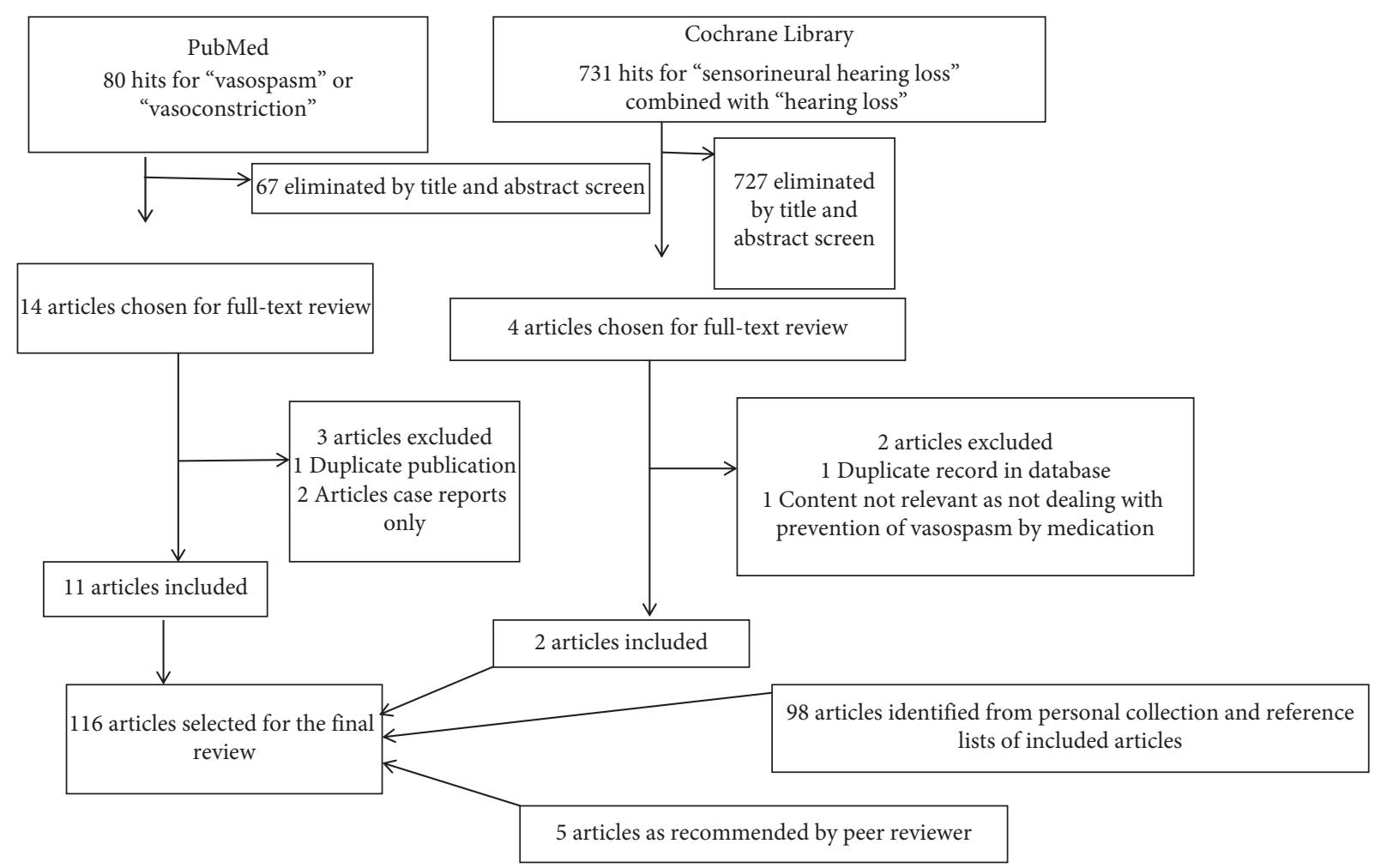

Figure 1: Flow chart of literature search.

Autoimmune diseases like systemic lupus erythematosus and Crohn's disease which induce overproduction of tumor necrosis factor (TNF) and rarer diseases like the cryopyrinassociated periodic syndromes with inflammasome activation and subsequent overproduction of interleukin-1(IL-1) like CINCA syndrome and Muckle-Wells disease have been associated with SNHL $[8,9]$. The blockage of TNF with antiTNF antibody constructs (for Crohn's disease) and anti-IL-1 (diseases with inflammasome activation) has been shown to reverse the hearing loss $[10,11]$. This is against what would be permanent damage to hair cells or neuronal damage involved in hearing loss or apoptosis and favours a reversible cause of immune-mediated hearing loss like vasospasm.

3.1.3. Bacterial Meningitis. In bacterial meningitis, severe inflammation of the meninges is observed which should, if there was a link between inflammation and SNHL lead to SNHL. Hearing loss is indeed observed in all forms of bacterial meningitis $[10,11]$.

In a prospective study of 236 children with meningitis using brainstem auditory evoked responses, 38 were detected with hearing loss in the acute (early) phase of meningitis; among these, 32 were available for follow-up, and within the latter group, 22 recovered normal hearing, i.e., reversibility was seen in $68.7 \%$ of the cases available for documentation. The hearing loss was unilateral in 21 of the 38 children with hearing loss detected in the acute meningitis phase $(=55.3 \%)$. The 21 unilateral HL cases among 236 children with bacterial meningitis amounted therefore to $9 \%[12]$.
An analysis of risk factors for SNHL in patients with bacterial meningitis identified focal neurological signs, as found in localized vasospasm, as an associated feature but not general severity of sepsis (see Table 1) [10]. Subsequent studies conducted in Malawi, Kenya, and Pakistan confirmed the association of SNHL with focal neurological deficits including cranial nerve palsies [13, 14]. A recent review article [1] highlighted that features of vasospasm were detected in the form of arterial stenosis in bacterial meningitis in a case of Haemophilus influenzae meningitis [15], whereas it was Greitz who was the first who detected evidence for vascular stenosis in the form of local vasoconstriction at the base of the brain in cases of Mycobacterium tuberculosis meningitis [16].

A prospective investigation of adults with bacterial meningitis by transcranial Doppler sonography related the degree of arterial narrowing to outcome including focal cerebral ischemic deficits, which were more frequent in patients with cerebral blood flow velocity $>210 \mathrm{~cm} / \mathrm{s}$ compatible with vasospasm [17].

Other investigations confirmed features of cerebral vasospasm in bacterial meningitis. Patients with features of vasospasm had significantly increased concentrations of IL-1 and IL-6 in the CSF. Underlying mechanisms have been explored in a rat model, where cerebral blood flow as measured by injection and recovery of microspheres from the brain tissue was found to be reduced in pneumococcal meningitis. The cerebral blood flow could be restored by the endothelin-1 antagonist bosentan with a reduction in cortical cerebral injury. Strong evidence of a protective role of the vasodilator nitric oxide against vasospasm in bacterial 
TABLE 1: Clinical manifestations, indicators of disease severity, and SNHL in bacterial meningitis (data from [10]).

\begin{tabular}{|c|c|c|c|c|}
\hline & $\begin{array}{l}\text { Cases with SNHL after bacterial meningitis } \\
\qquad(n=41)\end{array}$ & $\begin{array}{c}\text { Controls without SNHL after bacterial } \\
\text { meningitis }(n=129)\end{array}$ & $\begin{array}{c}P \\
\text { value }\end{array}$ & $\begin{array}{l}\text { Odds ratio } \\
(95 \% \mathrm{CI})\end{array}$ \\
\hline $\begin{array}{l}\text { Signs of shock on } \\
\text { admission }\end{array}$ & ( & 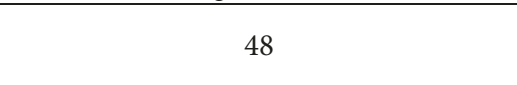 & 0.65 & $\begin{array}{c}0.78 \\
(0.35-1.76)\end{array}$ \\
\hline Mechanical ventilation & 2 & 16 & 0.24 & $\begin{array}{c}0.36 \\
(0.05-1.76)\end{array}$ \\
\hline Seizures & 10 & 29 & 0.96 & $\begin{array}{c}1.11 \\
(0.45-2.71)\end{array}$ \\
\hline Focal neurological signs & 16 & 9 & $<0.001$ & $9.0(3.11-27.7)$ \\
\hline
\end{tabular}

meningitis was derived from an animal model of neonatal meningitis where inhibition of NO synthase worsened outcome in some models of bacterial meningitis, leading to local cerebral ischemia $[18,19]$. The findings from these studies highlight that it is not simply the level of cytokines causing sepsis but a specific cytokine profile and the response of the body to it. Steroids have been shown to reduce the risk of development of SNHL in bacterial meningitis. They reduced the risk of death (RR: $0.46,95 \%$ CI: 0.24 to 0.88; 2 studies, $N=132$; very low-quality evidence) but did not have a significant effect on the number of infants with SNHL at two years (RR: $1.80,95 \%$ CI: 0.18 to 18.21 ; one study, $N=38$ participants, low-quality evidence). In one trial, dexamethasone reduced the likelihood of hearing loss at 4 to 10 weeks after discharge (RR: $0.41,95 \%$ CI: 0.17 to 0.98; one study, $N=59$ participants, low-quality evidence). There was no beneficial effect of corticosteroid therapy in low-income countries [20-22].

All these lines of evidence revealed features consistent with inflammation-associated vasospasm-induced SNHL but no direct evidence for a link between the hearing loss observed and vasospasm of blood supply of the cochlea.

3.1.4. Head or Brain Injury. A recent systematic review of 13 studies encompassing 773 patients found that the studies with the highest level of evidence reported a transient or chronic hearing loss of at least $10-15 \mathrm{~dB}$ across a range of frequencies in as many as $58 \%$ of patients with traumatic brain injury without bone fracture [22].

Vasospasm has hereby previously been associated with brain injury and was more severe in patients with associated features of a systemic inflammatory response [1].

In subarachnoid haemorrhage, vasospasm has been directly linked to hearing loss [23, 24]. The underlying mechanism appears to be free haemoglobin from the extravasated blood scavenging the vasodilator nitric oxide and inducing the vasoconstricting cytokine IL-1 via inflammasome activation. However, traumatic head injury is a condition associated with increased risk of inflammationassociated vasospasm-induced sensorineural hearing loss. Subarachnoid haemorrhage has features consistent with inflammation-associated vasospasm-induced sensorineural hearing loss.

A retrospective case control study of neonatal brain injury including 58 infants with SNHL and comparing them with 116 controls without hearing loss established that cerebral infarction was significantly increased in infants with
SNHL compared to normal hearing controls [25]. Cerebral infarction is a feature of vaso-occlusion and may be related to vasospasm in the neonatal period. Cerebral vasospasm may occur in association with intraventricular haemorrhages which were previously associated with vasospasminduced cerebral injury via haem released from haemoglobin, scavenging the vasodilator nitric oxide and stimulating production of the vasospasm-inducing interleukin-1 by inflammasome activation [26].

3.1.5. Noise-Induced Trauma. The labyrinthine artery is known to constrict during and after noise overstimulation [27]. TNF- $\alpha$ inhibition using etanercept prevented noiseinduced hearing loss by improvement of cochlear blood flow in vivo $[28,29]$. Prophylactic treatment with the calcium antagonist nimodipine was unsuccessful in prevention of noise-associated SNHL pointing to a calcium-independent effect of TNF on vasoconstriction [30]. For noise-induced hearing loss, there is therefore direct evidence for inflammation-associated vasospasm causing sensorineural hearing loss.

3.1.6. Presbyacusis. Age-related inflammatory diseases like type II diabetes and cardiovascular disease have been associated with SNHL [31, 32]. In 2012, the first investigation into a correlation of systemic inflammation with SNHL was undertaken in 611 people between 63 and 74 years of age and total white blood cell count, neutrophil count, and C-reactive protein (CRP) levels were found to be associated with SNHL independently of smoking, alcohol, and previous noise exposure. The authors did not forward any explanation for this finding [33]. The results of this study were confirmed in the "Epidemiology of Hearing Loss Study," which found an association of elevated CRP levels at baseline and future development of SNHL [34].

The theoretic possibility of vasospasm related to arteriosclerotic inflammation is raised by the transient audiovestibular dysfunction with transient hearing loss found as prodromal symptom of stroke affecting the supply area of the anterior inferior cerebellar artery in vertebrobasilar ischemic stroke affecting 8 to $31 \%$ of prospectively observed patients including patients with normal diffusion-weighted MRI after the attack [35]. Brown et al. [36, 37] found that cochlear vascular reactivity to topical application of nitroprusside, a vasodilating agent, was less in old mice than in young mice. 
Overall, the findings in presbyacusis justify the conjecture of an increased risk of inflammation-associated vasospasm-induced sensorineural hearing loss as a contributory underlying mechanism.

3.1.7. Migraine. The pain of migraine-associated headaches has been linked to the activation of nociceptors by the vasoconstriction-inducing cytokines TNF and IL-1 [38, 39]. The involvement of these two cytokines if they are involved in cytokine-induced and "vasospasm-related" SNHL would therefore make one expect that migraine is associated with "vasospasm-related" SNHL. Migraine has been associated with sudden and transient SNHL where there was cerebral infarction and children were affected [40-42] [43]. The findings are therefore overall showing features consistent with inflammation-associated vasospasm-induced sensorineural hearing loss.

3.2. Mechanisms Linking Cytokines to Vasoconstriction. Inflammatory mediator action could explain the role of vasospasm in the pathogenesis of SNHL in all the aforementioned conditions. For many years, the cochlea was considered an "immune-privileged" organ because of the presence of a tight junction-based blood-labyrinth barrier. A number of more recent studies, however, showed that resident macrophages are always present in the cochlear lateral wall as well as in the spiral limbus and the scala tympani side of the basilar membrane, and they are activated by various types of insults, including noise exposure, ischemia, mitochondrial damage, and surgical stress leading to cytokine release including tumour necrosis factor [44].

3.2.1. Tumor Necrosis Factor-Induced Vasospasm and Hearing Loss. TNF-alpha was shown to be effective in decreasing cochlear blood flow at a dose of $5.0 \mathrm{ng} / \mathrm{mL}$. Lower concentrations or placebo treatment did not lead to significant changes. After pretreatment with etanercept, a recombinant human TNF receptor p75 Fc fusion protein, which blocks TNF action, TNF-alpha at a dose of $5.0 \mathrm{ng} / \mathrm{mL}$, no longer led to a change in cochlear blood flow [45].

In a prospective clinical trial in Mongolian gerbils, tumor necrosis factor-alpha administered via continuous 8-day intrathecal flow induced SNHL across all frequencies compared to phosphate-buffered saline delivered intrathecally in controls and tumor necrosis factor-alpha antibody injected intraperitoneally reduced SNHL in the pneumococcal meningitis model [46].

In a prospective observational study, tumor necrosis factor-alpha levels in peripheral venous blood were compared between patients with autoimmune inner ear disease and sudden SNHL compared to controls and steroid responders compared with steroid nonresponders. TNF levels were significantly higher in patients with sudden SNHL, and a high baseline plasma TNF of greater than $18.8 \mathrm{pg} / \mathrm{ml}$ had a positive predictive value higher than $97 \%$ for a sudden change in hearing threshold [47] and was associated with a lack of response to steroids.
The mechanism by which tumor necrosis factor causes a reduction in cochlear blood flow has been identified and involved contraction of cochlear pericytes which has in the guinea pig model been found to cause a reduction of capillary diameter by $3.6 \pm 4.3 \%$, which was reversible by antiTNF therapy with ethanercept [48].

The effect of TNF on vasoconstriction may be mediated by sphingosine-1-phosphate (S1P) [49].

$\mathrm{S} 1 \mathrm{P}$ has been demonstrated to lead to vasoconstriction in cerebral and basilar arteries in vivo in animal models. This effect was shown to be mediated by elevation of intracellular calcium levels by mobilisation from intracellular stores in smooth muscle cells. $\mathrm{Ca}^{2+}$ sensitization of the contractile filaments, which frequently involves a rho-kinase, is also an effect of S1P and has been observed in human coronary smooth muscle cells. Inhibition of the rho A/rho-kinase pathway has hereby been shown to inhibit vasoconstriction [50]. It has also been established that the tone of the labyrinthine artery is regulated by S1P [51]. It has thus been shown that S1P could be involved in the pathogenesis of inflammation-induced hearing loss. TNF is also able to impair the stability of endothelial NO synthase mRNA, thus potentially undermining the bioavailability of the vasodilator NO [52].

The question is whether the chronic inflammation leading up to presbyacusis leads to SNHL via cytokine-induced apoptosis of hair cells of the inner ear or recurrent vasospasm in the labyrinthine artery. In addition to causing vasospasm, TNF could trigger programmed cell death (apoptosis) in hair cells of the inner ear and thus cause SNHL. The extrinsic pathway to apoptosis includes TNF alpha, which via TNF receptor-associated death domain activates cysteine-aspartic proteases (caspases)-8 and -10 . Caspase- 8 then can activate tBid to recruit the help of the intrinsic pathway and can activate caspases-3, -6 , and -7 . These caspases lead to the execution phase of cell death induction through their protein-cutting activity. Caspase-3 can hereby cause DNA and chromatin damage, rearrange the cytoskeleton, and disrupt intracellular transport [53]. Apoptosis-related hearing loss secondary to long-term exposure to circulating mediators of chronic inflammation including TNF should lead to bilateral SNHL. Studies on the effect of the anti-inflammatory drug dexamethasone (DXM) in vitro using 3-day-old rat organ of Corti explants challenged with tumor necrosis factor-alpha (TNFa) demonstrated the otoprotective capability of DXM against the ototoxic effects of this inflammatory cytokine on auditory hair cells. Cytokine-exposed explants treated with DXM prevented TNFa-induced loss of auditory hair cells and resulted in the downregulation of Bax gene expression with upregulation of both $\mathrm{Bcl}-2$ and $\mathrm{Bcl}-\mathrm{xl}$ gene expression compared with baseline levels of gene expression in control explants and with the expression levels of the housekeeping gene, $\beta$-actin. Biopolymer- (i.e., poly(styrene-b-isobutyleneb-styrene); SIBS) eluted DXMb retains its ability to prevent the death of explant auditory hair cells that were exposed to TNFa and to initiate the same patterns of upregulation and downregulation of antiapoptosis- and proapoptosis-related genes (i.e., Bcl-2, Bcl-xl, and $\mathrm{Bax}$ ) that were observed in 
TNFa-challenged and TNFa-challenged, DXM-treated organ of Corti explants [54].

3.2.2. IL-1 and Vasospasm-Associated SNHL. An investigation in patients with SAH showed that levels of IL-1 beta but not TNF-alpha were increased and correlated with the later development of vasospasm. IL-1 acts through G-protein-coupled receptors in three ways which cause contraction of vascular smooth muscle [1]:

(1) Activation of phospholipase C-generated phosphatidylinositol trisphosphate causes calcium release which leads to myosin light chain kinase activation leading to myosin light chain activation and calciumdependent vasospasm. Phosphorylation of myosin light chain (MLC) by MLC kinase is one of the most important steps for vascular smooth muscle contraction.

(2) Activation of protein kinase C (PKC) through phospholipase C-generated diacylglycerol (from phosphatidylinositol $(4,5)$ bisphosphate) can act through phosphorylation, hence activating the myosin light chain kinase. Prolonged contraction in cerebral vasospasm for up to two weeks can be mediated by this mechanism.

(3) PKC activation regulates MLC phosphorylation through activation of rho-kinase and the myosinbinding subunit (MBS) of MLC phosphatase (MLCPh).

A secondary pathway for vascular smooth muscle contraction that is not directly dependent on $\mathrm{Ca}^{2+}$ concentration, but rather mediating $\mathrm{Ca}^{2+}$ sensitization, is the rho A/rho-kinase pathway. In cultured human coronary vascular smooth muscle cells, inflammatory stimuli, such as IL-1 beta, increase rho-kinase expression (at both mRNA and protein levels) and function [55].

Both endothelial NO production and NO-mediated signaling in vascular smooth muscle cells (VSMC) are targets and effectors of the rho $\mathrm{A} /$ rho-kinase pathway. In endothelial cells (ECs), the rho A/rho-kinase pathway negatively regulates NO production [56]. In addition, in response to contractile stimuli, the small GTPase rho A activates its downstream effector rho-kinase which, in turn, promotes contraction via myosin light chain phosphatase (MLCP) inhibition. Rho-kinase activity is enhanced by binding to the active GTP-bound rho A. Experiments were performed to examine whether rho-kinase is upregulated at the spastic site and how it induces VSMC hypercontraction if it is upregulated. Reverse transcriptase polymerase chain reaction analysis demonstrated that the expression of rhokinase mRNA and, to a lesser extent, that of RhoA mRNA were upregulated in the spastic site than the control coronary site. Rho A/rho-kinase-mediated MLCP inhibition occurs mainly by phosphorylation and inhibition of MYPT1 , the regulatory subunit of MLCP, or by CPI-17-mediated inhibition of the catalytic subunit of MLCP. Rho-kinase hereby phosphorylates MBS, which results in the inhibition of MLCPh [57]. These results indicate that rho-kinase is upregulated at the spastic site and plays a key role in inducing VSMC hypercontraction by inhibiting MLCP through MYPT-1 phosphorylation. The reduced MLCPh activity leads to increased phosphorylation and hence contractility of the MLC resulting in calcium-independent vasospasm (See Figure 2). Investigations in a porcine model revealed that hydroxyfasudil, a specific rho-kinase inhibitor, exerted an inhibitory effect on vasospasm both in vitro and in vivo. Western blot analysis showed that, during serotonin-induced contractions, the extent of phosphorylation of the MBS was significantly greater in the spastic than in the control segment. There was a highly significant correlation between the extent of MBS phosphorylation and contractions. The potential of fasudil in prevention and treatment of vasospasm-associated SNHL was demonstrated in an experiment with the small modiolar artery isolated from temporal bone of gerbils in which it prevented endothelin-1induced contraction and $\mathrm{Ca}^{2+}$ sensitization [58].

Endothelin-1 is one way of action of both TNF and IL-1 on vasospasm-induced hearing loss. Only ET-1 is produced in cerebral endothelium and mediates cerebral vasoconstriction via endothelin-A (ETA) receptors. ETA is localized in vascular smooth muscle cells, and stimulation leads to increase in intracellular calcium concentrations leading to vasoconstriction. ET-1 expression is enhanced by IL-1 and TNF and can be inhibited by NO, nitric oxide donor drugs, and dilator prostanoids via an increase in cellular cGMP. NO can produce relaxation of some blood vessels from some species via activation of potassium channels [1].

3.2.3. Cytokine Gene Polymorphisms and SNHL. Should cytokines have a role in SNHL one would expect that cytokine gene polymorphisms which are associated with differences in cytokine production differ between patients prone to hearing loss and those who are not. This is in fact the case.

A significant difference in carriage of both the IL$1 \beta-511 \mathrm{~T}$ allele and the IL- $\beta+3953 \mathrm{~T}$ allele was observed between sudden SNHL and controls. This suggests that the IL- $1 \beta-511$ and +3953 loci may play an important role in the etiopathogenesis of SSNHL [59].

Genetic linkage studies have investigated the association of polymorphisms of the TNF-alpha and TNF-beta genes with sudden SNHL comparing 97 SSNHL patients and 587 controls and found that the TNF-beta $+252 \mathrm{~A}-\longrightarrow \mathrm{G}$ polymorphism which is associated with increase in production of this cytokine was significantly increased in patients with SSNHL [60], a locus, which had previously been linked to cerebral infarction [61].

\subsection{Treatment and Prevention of Vasospasm-Induced SNHL}

\subsubsection{Calcium Antagonists}

(1) Calcium-Channel Blockers. As illustrated in anti-phospholipid antibody syndrome, an autoimmune inflammatory syndrome, which is associated with cerebral vasoconstriction 


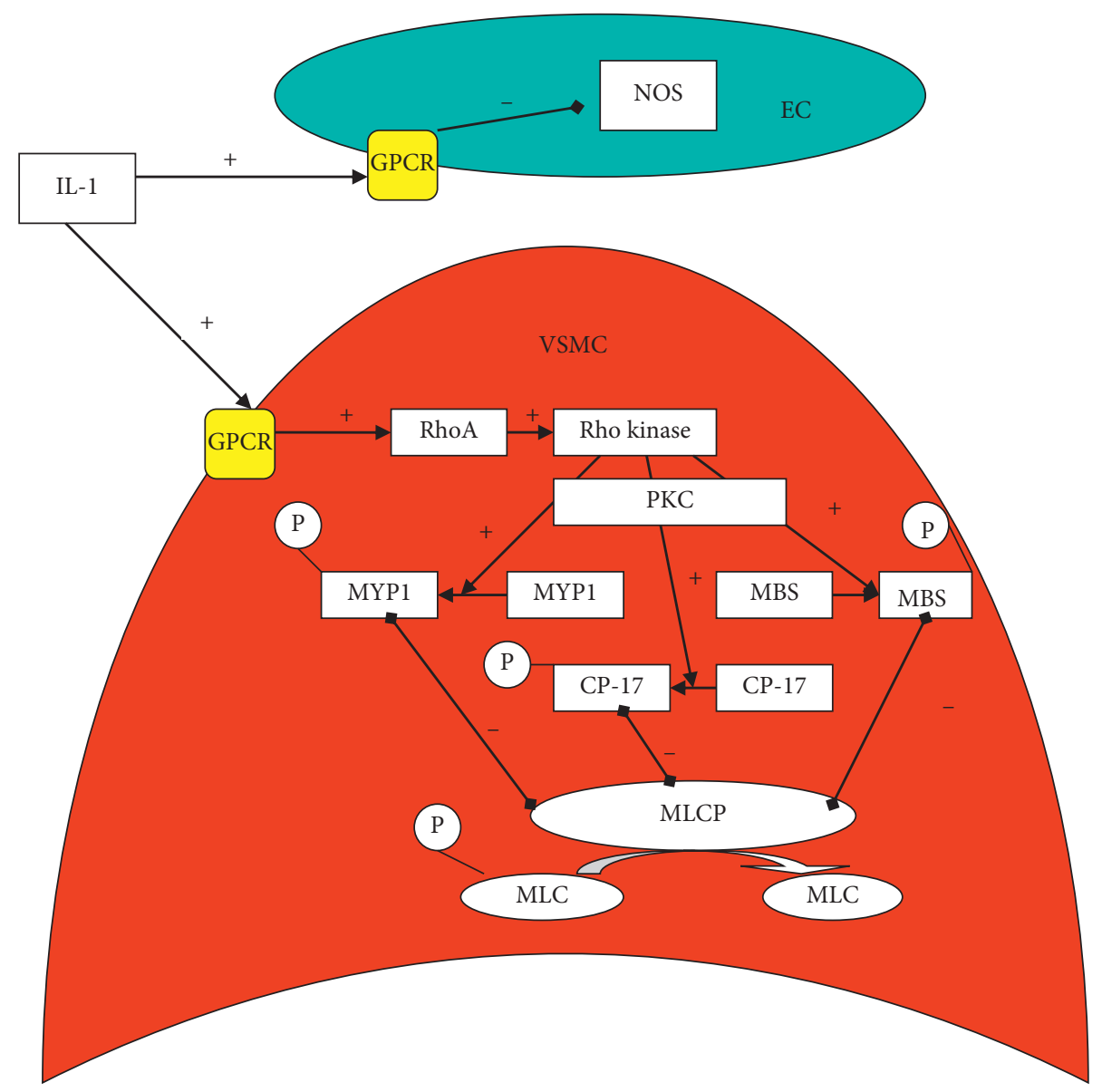

FIGURE 2: Mechanism of action of interleukin-1 (IL-1) via G-protein coupled receptors (GPCR) on reduction of nitric oxide (NO) production by reducing nitric oxide synthase (NOS) activity in the vascular endothelial cell (EC) and by activation of the enzyme Rho kinase via RhoA in the vascular smooth muscle cell to activate by phosphorylation through protein kinase $\mathrm{C}$ (PKC) myosine phosphatase targeting subunit-1 (MYPT1), protein kinase C-potentiated phosphatase inhibitor-17 (CPI-17 and myosine binding subunit (MBS)), which all three inhibit the myosine light chain phosphatase (MLCP) which dephosphorylates the myosine light chain, a process, which is necessary for smooth muscle relaxation. This process thus causes vasoconstriction.

syndrome, centrally acting calcium antagonists like nimedipine, can reduce symptoms of cerebral vasoconstriction, which include hearing loss [62].

Blockade of L-type voltage-gated $\mathrm{Ca}^{2+}$ channels protected against NIHL in mice and in guinea pigs, and blockade of T-type voltage-gated $\mathrm{Ca}^{2+}$ channels was effective in mice. Also consistent with a contribution of calcium-mediated events in hair cell damage, application of the calcineurin inhibitor FK506 attenuated NIHL in guinea pigs [63-66].

(2) Magnesium. An agent that can lead to vasodilatation by virtue of antagonism of calcium-dependent vascular smooth muscle contraction is magnesium. It seems to act through a variety of pathways. Experiments in laboratory animals demonstrated that as magnesium decreases, there is a greater secretion of catecholamines and prostaglandins, subsequently leading to a reduction of the blood flow because of vasoconstriction in the inner ear and with it, a higher risk of energetic depletion in the hair cells. Increased magnesium intake can thereby improve inner ear microcirculation. The muscle tone of the vessels diminishes with higher concentrations of intracellular free magnesium, and their reactivity to vasoactive substances decreases [67-69].

In addition to the well-characterized effects on vasodilation, biochemical effects of magnesium include modulation of calcium channel permeability, influx of calcium into cochlear hair cells, and glutamate release [70, 71].

The first evidence for the importance of magnesium in prevention of SNHL was gained in observational studies of magnesium deficiency in rats and guinea pigs exposed to noise. Magnesium deficiency increased susceptibility to NIHL in rats [72] and guinea pigs [73]. Subsequent experiments then confirmed the protective effect of magnesium in prevention of SNHL in the guinea pig model [74-76].

The results of magnesium in animal experiments have have been confirmed in 3 randomised controlled trials [77-79] summarized in Table 2 which confirmed their applicability in humans and that magnesium has the potential to prevent noise-induced SNHL in humans. When measured it was not serum magnesium levels, which were different between groups, but the magnesium levels in red blood cells and monocytes or lymphocytes [78, 79]. 


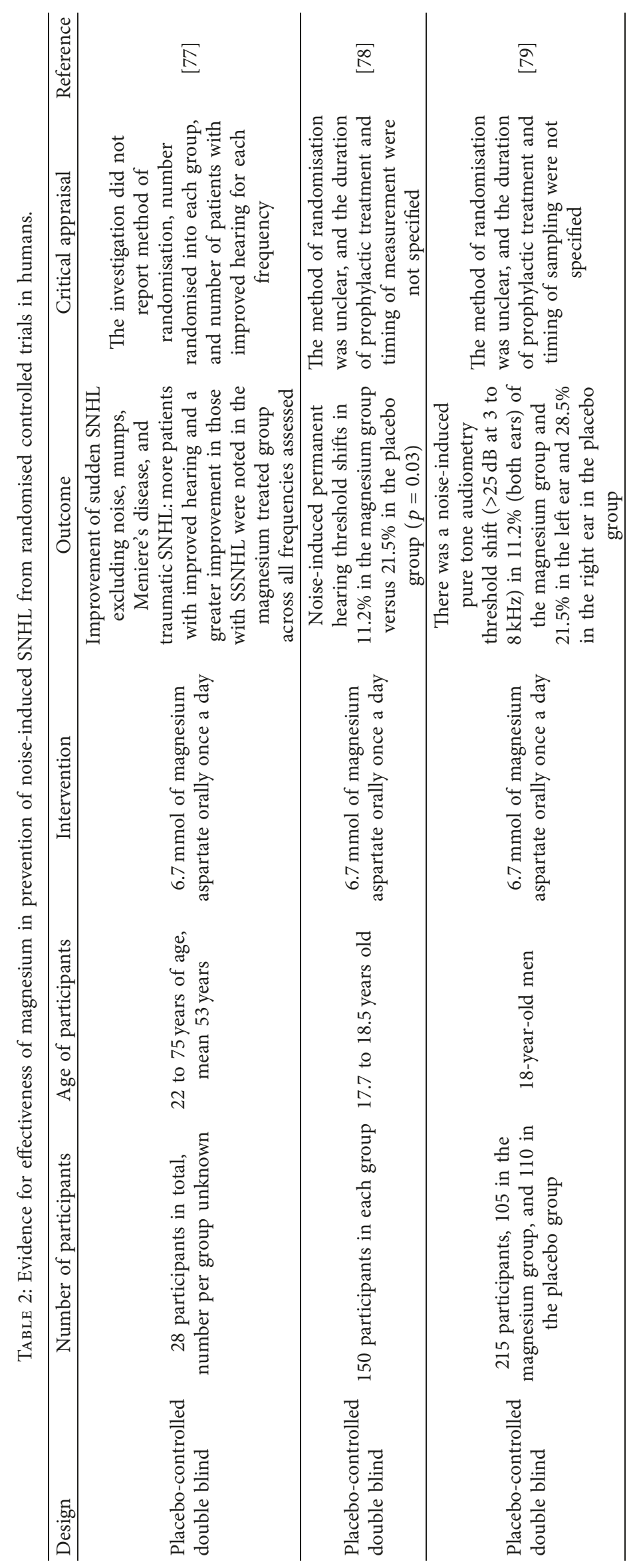




\subsubsection{Anti-Inflammatory Therapies}

(1) Etanercept. Etanercept is a widely used biological agent in the treatment of autoimmune diseases such as rheumatoid arthritis, psoriasis, and others by targeting TNF- $\alpha$. This and other anticytokine therapies, which show promise in prevention or treatment of inflammation-induced SNHL, have been reviewed previously [80], and the results of this review were that etanercept can mitigate the inflammation and hearing loss (HL) in labyrinthitis induced by keyhole limpet hemocyanin (KLH) in mice. In a guinea pig model of immune-mediated HL, within 3-5 days of KLH insult, the labyrinth developed features of inflammation associated with HL. Both the systemic treatment with etanercept and long-term infusion of etanercept (for 7 days) into the scala tympani significantly protected the labyrinth against KLHinduced inflammation and the development of HL. Systemic administration of etanercept after loud noise exposure (106 dB SPL, $30 \mathrm{~min})$ significantly attenuated NIHL. The microcirculatory analysis of cochlea showed significant increase in cochlear blood flow in strial capillary segments. Moreover, significant hearing improvement was observed in etanercept-treated groups versus controls. The TNF- $\alpha$ inhibitor etanercept has hereby been shown to reduce noiseinduced threshold shifts [81]. A very small randomised controlled trial in autoimmune SNHL in humans did not show any benefit, but the small sample size ( $n=17$ in total) means that a clinically significant difference may have been missed [82].

(2) Steroids. The anti-inflammatory and proresolution glucocorticoid dexamethasone (DEXA), which is able to reduce TNF production, has been investigated after systemic, intratympanic, and combined delivery in sudden and noiseinduced SNHL but with mixed results as shown in the following four meta analyses [83-86] (Table 3).

(3) Statins. Statins are inhibitors of the 3-hydroxy-3-methylglutaryl-coenzyme A reductase, which is a key enzyme of the mevalonate pathway, the metabolic pathway that produces cholesterol and other isoprenoids. It also has a direct effect on rho-kinase activity and hence the predisposition to vasospasm. This effect is mediated through several different mechanisms: because statins inhibit an early step in the cholesterol biosynthetic pathway, they also inhibit the synthesis of isoprenoids such as farnesylpyrophosphate (FPP) and geranylgeranylpyrophosphate (GGPP), which are important posttranslational lipid attachments for intracellular signaling molecules such as the rho GTPases. Treatment with statins prevents endothelial nitric oxide synthase (eNOS) downregulation by prolonging the half-life of eNOS mRNA. The prolongation of half-life of eNOS mRNA by statins is reversed by GGPP, but not FPP, suggesting the involvement of small GTPases such as rho GTPase in this process. Indeed, decrease in rho GTPase responses as a consequence of statin treatment increases the production and bioavailability of endothelium-derived NO. The mechanism involves, in part, rho/rho-kinase- (ROCK-) mediated changes in the actin cytoskeleton, which leads to decreases in eNOS mRNA stability. In addition to increase in eNOS expression by statins, statins can also rapidly induce the phosphorylation and activation of eNOS via the phosphatidylinositol-3 kinase (PI3K)/protein kinase Akt pathway [87]. Inhibition of mevalonate synthesis by statins decreases geranylgeranyl pyrophosphate and prevents the geranylgeranylation of rho and therefore its activation of rho-kinase (ROCK) [88]. It has been reported that atorvastatin reduces inducible nitric oxide synthase expression in native endothelial cells in situ and the underlying mechanism is associated with inhibition of tumor necrosis factor- $\alpha$ and interferon- $\gamma$ expression [89]. In rats, atorvastatin was able to prevent noise-induced SNHL [90]. The same was found in mice [91] and more recently in guinea pigs [92]. In a prospective study of 84 hyperlipidemic patients and audiological assessment before and 6 months after commencement of a statin, a significant reduction of the hearing threshold at $6000 \mathrm{~Hz}$ was found and a significantly improved speech discrimination as well as a significant reduction in tinnitus frequency, duration, and severity and degree of annoyance [93].

This result confirmed a previous study: The Blue Mountains Hearing Study is a population-based survey of age-related hearing loss which aimed to assess associations between dietary intake of fats (saturated and monounsaturated fats and cholesterol) and certain food groups (butter, margarine, and nuts) with the prevalence, incidence, and progression of age-related hearing loss. It also investigated the link between serum lipids and cholesterol-lowering medication (statins) and hearing loss. Among persons self-reporting statin use $(n=274)$, a $48 \%$ reduced odds of prevalent hearing loss was observed after multivariable adjustment $\quad(\mathrm{OR}=0.52 \quad(95 \%$ $\mathrm{CI}=0.29-0.93))[94]$.

On the other hand, a population-based study in Taiwan showed a slight but significant association of previous statin use and SNHL ( $27 \%$ with versus $21 \%$ without previous statin use, OR 1.36 (95\% CI: 1.18 to 1.57$)$ ) [95].

The only randomised double-blind clinical trial in this area was geared to investigate the impact of statins on presbyacusis. It was very small with a total of 50 patients. Pure-tone audiometry and tinnitus evaluation at enrolment and after 7 and 13 months of atorvastatin did not show any impact on hearing thresholds or tinnitus [96].

(4) Oral Curcumin. Curcumin, a compound isolated from the plant Curcuma longa, is the principal curcuminoid of turmeric, a member of the ginger family. It has been shown to reduce vascular inflammation and the related vasospasm in cerebral blood vessels in animal models of subarachnoid haemorrhage [97, 98].

It is therefore not surprising that oral curcumin in a highly bioavailable preparation has been shown to reduce noise-induced SNHL in a murine model in a dose-dependent fashion [99].

One mechanism may be its capacity for reduction of TNF expression [100] which is probably mediated by inhibition of epigenetic activation of cytokine expression through inhibition of histone deacetylase expression [101]. 


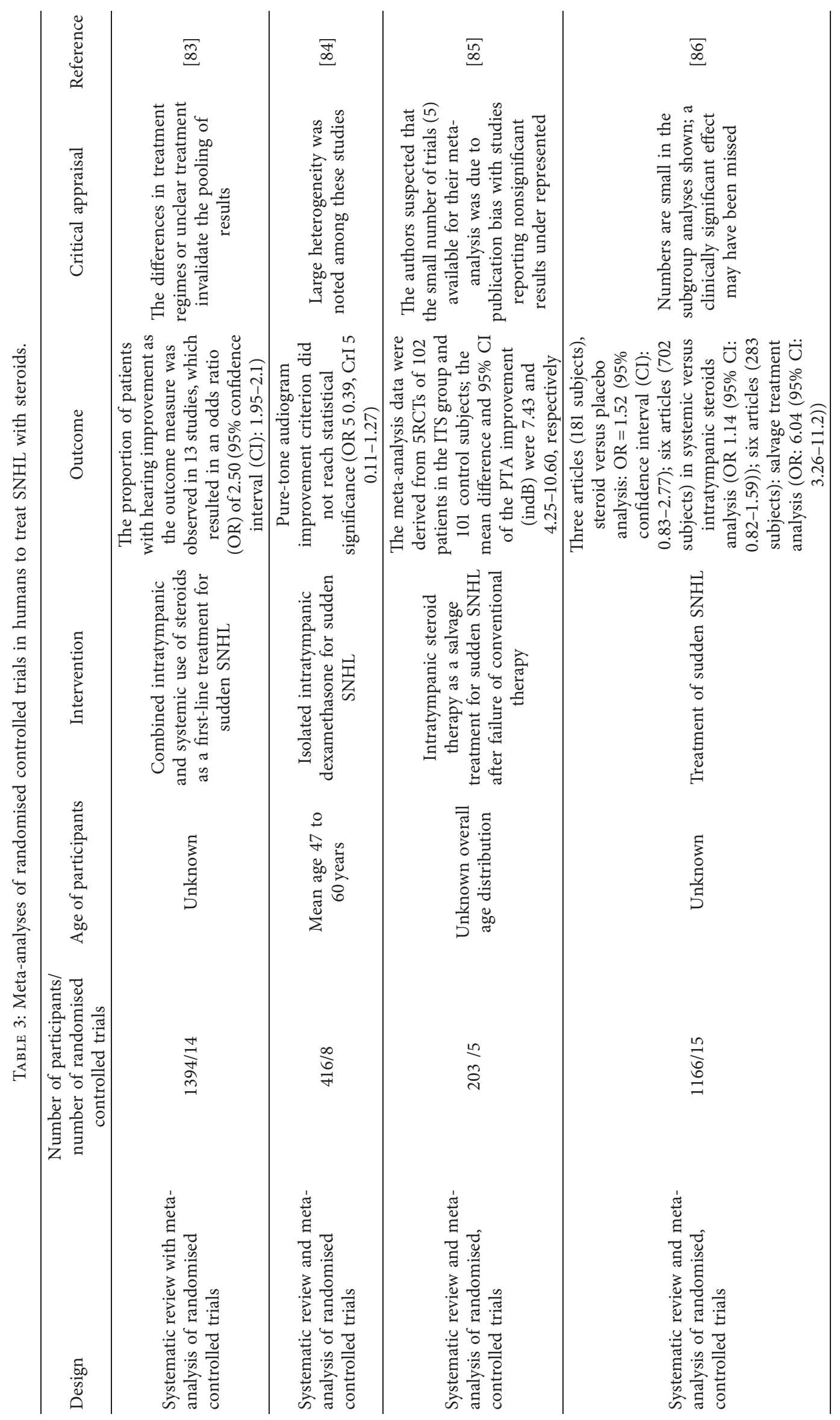


TABLE 4: Directness of evidence for a role of inflammation-induced vasospasm in conditions associated with acquired SNHL.

\begin{tabular}{lcccc}
\hline Condition associated with SNHL & $\begin{array}{c}\text { Evidence for } \\
\text { vasospasm }\end{array}$ & $\begin{array}{c}\text { Evidence of link of } \\
\text { SNHL to vasoconstrictors }\end{array}$ & $\begin{array}{c}\text { Evidence for response to } \\
\text { anti-inflammatory treatment }\end{array}$ & $\begin{array}{c}\text { Evidence for treatment } \\
\text { response to vasodilators }\end{array}$ \\
\hline Autoimmune & Features & Features & Features & Not investigated \\
Bacterial meningitis & Features & Features & Features & Features \\
Phonic injury & Direct & Direct & Direct & Not investigated \\
Subarachnoid haemorrhage & Features & Features & Features & Not investigated \\
Brain trauma & Increased risk & No evidence & No evidence & Not investigated \\
Presbyacusis & No evidence & No evidence & No evidence & Not investigated \\
Migraine & Features & Not investigated & Not investigated & Direct \\
Sudden SNHL & Direct & Not investigated & Direct &
\end{tabular}

(5) Phosphodiesterase-5 Inhibitors. Phosphodiesterase-5 inhibitors are potent vasodilators and can relieve vasospasm. It is therefore conceivable that they could reduce or prevent inflammatory vasospasm-related hearing loss. The mechanism of action involves causing increased bioavailability of cGMP, a potent endogenous vasodilator: When nitric oxide (NO) forms in the body, it avidly binds to the heme moiety of the enzyme guanylyl cyclase, which is found in vascular smooth muscle cells. NO is formed by vascular endothelium and diffuses into the vascular smooth muscle cells adjacent to the endothelium where it binds to and activates guanylyl cyclase. This enzyme catalyzes the dephosphorylation of GTP to cGMP, which serves as a second messenger particularly for signaling smooth muscle relaxation. Cyclic GMP induces smooth muscle relaxation by multiple mechanisms including

(1) Increasing intracellular cGMP, which inhibits calcium entry into the cell, and decreasing intracellular calcium concentrations

(2) Activating $\mathrm{K}^{+}$channels, which leads to hyperpolarization and relaxation

(3) Stimulating a cGMP-dependent protein kinase that activates myosin light chain phosphatase, the enzyme that dephosphorylates myosin light chains, which leads to smooth muscle relaxation

Because of the central role of cGMP in NO-mediated vasodilation, drugs that inhibit the breakdown of cGMP (cGMP-dependent phosphodiesterase inhibitors) are used to enhance NO-mediated vasodilation.

Experiments in the mouse model of noise-induced SNHL demonstrated that Pde5 inhibition protected against NIHL [102]. Pde5 inhibition has also been clinically beneficial in reversing deficits in stroke patients [103].

There have been concerns about an association of phosphodiesterase- 5 inhibitor use and SNHL but this can be attributed to a confounding association of erectile dysfunction and hearing loss. Erectile dysfunction is the most common indication for phosphodiesterase-5 inhibition. Erectile dysfunction is liked to SNHL because both can be due to vascular dysfunction or vasospasm in the supplying artery in arteriosclerosis [104-107].

\section{Discussion}

Inflammation is a normal adaptive response aimed at restoring tissue functionality and homeostasis after infection and tissue injury, and suppressing it could have unintended negative consequences. Previous authors recognized the potentially important role of inflammation in causing age, noise, and drug-induced acquired SNHL but merely proposed to reduce the inflammatory response as a strategy to reduce SNHL [44]. This approach may not be rapidly and comprehensively effective if only directed at cochlear inflammation and not taking into account the importance of rapidly reversing vasospasm in order to reduce ischemic damage to the hair cells and other vital structures of the cochlea including targeting the specific mediators involved in vasospasm.

The evidence for a link between inflammation-induced vasospasm and acquired SNHL revealed in this review can be categorized as indicating a risk, as showing features or directness for the conditions identified above (see Table 4):

In conditions like bacterial meningitis, there is some hearing loss and there is some indirect evidence that there is vasospasm in bacterial meningitis. Further research needs to link vasospasm with hearing loss in prospective observational studies focussing on cochlear blood supply.

Similarly in traumatic brain injury, there is hearing loss in brain injury and there is vasospasm in brain injury, but the association between brain injury and vasospasm-mediated SNHL is only inferred from these two separate sets of findings, without being actually proven in any study.

Inhibition of rho-associated kinases by agents like fasudil needs to be explored in all patients at risk of vasospasm associated hearing loss or with established acquired SNHL [108, 109].

Future studies need to explore substances known to increase cochlear blood flow in ex vivo experiments, and these include herbal drugs used by traditional herbal medicine to treat hearing loss like Ginkgo biloba-derived quercetin, rosmarinic acid, and curcumin, the application of which has been shown to dilate cochlear vasculature and improve cochlear blood flow as measured by Doppler flowmetry in the guinea pig ex vivo model $[110,111]$.

Tinnitus has been associated with vasospasm in subarachnoid haemorrhage [112] and migraine has been associated with both vasospasm and tinnitus [41, 42], and vertigo is associated with SNHL in vascular compromise [113].

Vascular inflammation may not just be reduced in the labyrinthine artery by the modes of treatment explored in this review but other vascular beds as well, and future randomised controlled trials should incorporate other vestibular disorders including vertigo and tinnitus and vascular headaches as secondary outcomes as well as heart 
disease, stroke, vascular dementia, peripheral vascular disease, and their incidence could be monitored during long term follow-up of patients enrolled.

\section{Conclusions}

There is mainly indirect evidence for vasospasm-associated SNHL in most forms of systemic or injury- or infectioninduced local vascular inflammation. This opens up avenues in prevention and treatment of vascular and systemic inflammation as well as vasospasm itself as a way to prevent and treat most forms of acquired SNHL. Future research needs to investigate interventions antagonising vasospasm and vasospasm-inducing proinflammatory cytokines and their production in randomised controlled trials of prevention and treatment of acquired SNHL. Prime candidates for interventions are hereby inflammasome inhibitors and vasospasm-reducing drugs like nitric oxide donors, rhokinase inhibitors and magnesium which have the potential to reduce SNHL in meningitis, exposure to noise, brain injury, arteriosclerosis, and advanced age-related and autoimmune disease-related inflammation.

\section{Abbreviations}

AICA: Anterior inferior cerebellar artery

GPCR: G-protein coupled receptors

CD: $\quad$ Capillary density

CPI-17: Protein kinase C-potentiated phosphatase inhibitor-17

FPP: Farnesylpyrophosphate

GGPP: Geranylgeranylpyrophosphate

HL: $\quad$ Hearing loss

IL-1: Interleukin-1

MBS: Myosine-binding subunit

MLC: $\quad$ Myosine light chain

MLCPh: Myosine light chain phosphatase

MYPT1: Myosine phosphatase targeting subunit-1

NIHL: Noise-induced hearing loss

NO: $\quad$ Nitric oxide

NOS: $\quad$ Nitric oxide synthase

PKC: $\quad$ Protein kinase $\mathrm{C}$

PORH: Postocclusive reactive hyperaemia

S1P: $\quad$ Sphingosine-1-phosphate

SNHL: Sensorineural hearing loss

TNF: Tumor necrosis factor.

\section{Conflicts of Interest}

The author declares that there are no conflicts of interest involved in the publication of this article.

\section{References}

[1] M. Eisenhut, "Vasospasm in cerebral inflammation," International Journal of Inflammation, vol. 2014, Article ID 509707, 14 pages, 2014.

[2] M. Eisenhut, "In diabetic ketoacidosis brain injury including cerebral oedema and infarction is caused by interleukin-1," Medical Hypotheses, vol. 121, pp. 44-46, 2018.
[3] F.-J. Li, D.-Y. Wang, H.-Y. Wang et al., "Clinical study on 136 children with sudden sensorineural hearing loss," Chinese Medical Journal, vol. 129, no. 8, pp. 946-952, 2016.

[4] Y. Qian, S. Zhong, G. Hu, H. Kang, L. Wang, and Y. Lei, "Sudden sensorineural hearing loss in children," Otology \& Neurotology, vol. 39, no. 8, pp. 1018-1024, 2018.

[5] H. E. Koçak, A. Ş. Filiz Acıpayam, H. Acıpayam et al., "Microvascular dysfunction affects the development and prognosis of sudden idiopathic hearing loss," Clinical Otolaryngology, vol. 42, no. 3, pp. 602-607, 2017.

[6] T. Mom, A. Montalban, T. Khalil et al., "Vasospasm of labyrinthine artery in cerebellopontine angle surgery: evidence brought by distortion-product otoacoustic emissions," European Archives of Oto-Rhino-Laryngology, vol. 271, no. 10, pp. 2627-2635, 2014.

[7] J. J. Digiovanni and P. Nair, "Spontaneous recovery of sudden sensorineural hearing loss: possible association with autoimmune disorders," Journal of the American Academy of Audiology, vol. 17, no. 7, pp. 498-505, 2006.

[8] H. Staecker and P. P. Lefebvre, "Autoimmune sensorineural hearing loss improved by tumor necrosis factor- $\alpha$ blockade: a case report," Acta Oto-Laryngologica, vol. 122, no. 6, pp. 684-687, 2002.

[9] A. Vambutas and S. Pathak, "AAO: autoimmune and autoinflammatory (disease) in otology: what is new in immune-mediated hearing loss," Laryngoscope Investigative Otolaryngology, vol. 1, no. 5, pp. 110-115, 2016.

[10] M. Eisenhut, T. Meehan, and S. D. G. Stephens, "Risk factors for hearing loss in bacterial meningitis: delay in treatment and clinical manifestations," Journal of Audiological Medicine, vol. 11, no. 2, pp. 86-97, 2002.

[11] M. Eisenhut, T. Meehan, and L. Batchelor, "Cerebrospinal fluid glucose levels and sensorineural hearing loss in bacterial meningitis," Infection, vol. 3, no. 4, pp. 247-250, 2003.

[12] H. Guiscafré, M. C. Martínez, L. Benitez-Díaz, and O. Muñoz, "Reversible hearing loss after meningitis," Annals of Otology, Rhinology and Laryngology, vol. 93, no. 3, pp. 229-232, 1984.

[13] H. Forsyth, F. Kalumbi, E. Mphaka et al., "Hearing loss in Malawian children after bacterial meningitis: incidence and risk factors," Audiological Medicine, vol. 2, no. 2, pp. 100-107, 2009.

[14] B. W. Karanja, H. O. Oburra, P. Masinde, and D. Wamalwa, "Risk factors for hearing loss in children following bacterial meningitis in a tertiary referral hospital," International Journal of Otolaryngology, vol. 2013, Article ID 354725, 2013.

[15] E. L. Lyons and N. E. Leeds, "The angiographic demonstration of arterial vascular disease in purulent meningitis," Radiology, vol. 88, no. 5, pp. 935-938, 1967.

[16] T. Greitz, "Angiography in tuberculous meningitis," Acta Radiologica, vol. 2, no. 5, pp. 369-378, 1964.

[17] S. Ries, U. Schminke, K. Fassbender, M. Daffertshofer, W. Steinke, and M. Hennerici, "Cerebrovascular involvement in the acute phase of bacterial meningitis," Journal of Neurology, vol. 244, no. 1, pp. 51-55, 1996.

[18] L. A. Pfister, J. H. Tureen, S. Shaw et al., "Endothelin inhibition improves cerebral blood flow and is neuroprotective in pneumococcal meningitis," Annals of Neurology, vol. 47, no. 3, pp. 329-335, 2000.

[19] S. L. Leib, Y. S. Kim, S. M. Black, J. H. Tureen, and M. G. Täuber, "Inducible nitric oxide synthase and the effect of aminoguanidine in experimental neonatal meningitis," The Journal of Infectious Diseases, vol. 177, no. 3, pp. 692700, 1998. 
[20] M. C. Brouwer, P. McIntyre, K. Prasad, and D. van de Beek, "Corticosteroids for acute bacterial meningitis," Cochrane Systematic Review, 2015.

[21] T. A. Ogunlesi, C. C. Odigwe, and O. T. Oladapo, "Adjuvant corticosteroids for reducing death in neonatal bacterial meningitis," Cochrane Systematic Review, 2015.

[22] J. X. Chen, M. Lindeborg, S. D. Herman et al., "Systematic review of hearing loss after traumatic brain injury without associated temporal bone fracture," American Journal of Otolaryngology, vol. 39, no. 3, pp. 338-344, 2018.

[23] R. Ramdasi, A. Chagla, and A. Mahore, "Vasospasm causing reversible cortical deafness in subarachnoid hemorrhage," Neurology India, vol. 62, no. 4, pp. 469-470, 2014.

[24] S. Tabuchi, M. Kadowaki, and T. Watanabe, "Reversible cortical auditory dysfunction caused by cerebral vasospasm after ruptured aneurysmal subarachnoid hemorrhage and evaluated by perfusion magnetic resonance imaging," Journal of Neurosurgery, vol. 107, no. 1, pp. 161-164, 2007.

[25] S. Coenraad, A. Goedegebure, J. B. van Goudoever, and L. J. Hoeve, "Risk factors for sensorineural hearing loss in NICU infants compared to normal hearing NICU controls," International Journal of Pediatric Otorhinolaryngology, vol. 74, no. 9, pp. 999-1002, 2010.

[26] M. Eisenhut and S. Choudhary, "In premature newborns intraventricular hemorrhage causes cerebral vasospasm and associated neurodisability via heme-induced inflammasomemediated interleukin-1 production and nitric oxide depletion," Frontiers of Neurology, vol. 8, no. 423, 2017.

[27] Y. Nakai and H. Masutani, "Noise-induced vasoconstriction in the cochlea," Acta Oto-Laryngologica, vol. 105, no. sup447, pp. 23-27, 1988.

[28] C.-H. Choi, "Mechanisms and treatment of blast induced hearing loss," Korean Journal of Audiology, vol. 16, no. 3, pp. 103-107, 2012.

[29] W. Arpornchayanon, M. Canis, F. Ihler, C. Settevendemie, and S. Strieth, "TNF- $\alpha$ inhibition using etanercept prevents noise-induced hearing loss by improvement of cochlear blood flow in vivo," International Journal of Audiology, vol. 52, no. 8, pp. 545-552, 2013.

[30] F. A. Boettcher, R. K. Caldwell, M. A. Gratton, D. R. White, and L. R. Miles, "Effects of nimodipine on noise-induced hearing loss," Hearing Research, vol. 121, no. 1-2, pp. 139-146, 1998.

[31] P. Mitchell, B. Gopinath, C. M. McMahon et al., "Relationship of type 2 diabetes to the prevalence, incidence and progression of age-related hearing loss," Diabetic Medicine, vol. 26, no. 5, pp. 483-488, 2009.

[32] G. A. Gates, J. L. Cobb, R. B. D’Agostino, and P. A. Wolf, "The relation of hearing in the elderly to the presence of cardiovascular disease and cardiovascular risk factors," Archives of Otolaryngology-Head and Neck Surgery, vol. 119, no. 2, pp. 156-161, 1993.

[33] C. A. Verschuur, A. Dowell, H. E. Syddall et al., "Markers of inflammatory status are associated with hearing threshold in older people: findings from the Hertfordshire ageing study," Age and Ageing, vol. 41, no. 1, pp. 92-97, 2012.

[34] S. D. Nash, K. J. Cruickshanks, W. Zhan et al., "Long-term assessment of systemic inflammation and the cumulative incidence of age-related hearing impairment in the epidemiology of hearing loss study," The Journals of Gerontology: Series A, vol. 69A, no. 2, pp. 207-214, 2014.

[35] H.-A. Kim and H. Lee, "Recent advances in understanding audiovestibular loss of a vascular cause," Journal of Stroke, vol. 19, no. 1, pp. 61-66, 2017.
[36] J. N. Brown and A. L. Nuttall, "Autoregulation of cochlear blood flow in Guinea pigs," The American Journal of Physiology, vol. 266, pp. 458-467, 1994.

[37] J. N. Brown, J. M. Miller, and A. L. Nuttall, “Age-related changes in cochlear vascular conductance in mice," Hearing Research, vol. 86, pp. 189-194, 1995.

[38] I. Munno, N. M. Pellegrino, C. Marcuccio, L. Conrotto, E. Jirillo, and V. Covelli, "Neurological damage mediated by cytokines," Acta Neurologica (Napoli), vol. 14, no. 2, pp. 81-89, 1992.

[39] V. Covelli, L. Munno, N. M. Pellegrino et al., "Are TNF-alpha and IL-1 beta relevant in the pathogenesis of migraine without aura?," Acta Neurologica (Napoli), vol. 13, no. 2, pp. 205-221, 1991.

[40] A. F. Lipkin, H. A. Jenkins, and N. J. Coker, "Migraine and sudden sensorineural hearing loss," Archives of Otolaryngology-Head and Neck Surgery, vol. 113, no. 3, pp. 325-326, 1987.

[41] R. W. Evans and G. Ishiyama, "Migraine with transient unilateral hearing loss and tinnitus," Headache: The Journal of Head and Face Pain, vol. 49, no. 5, pp. 756-758, 2009.

[42] H. Lee, G. T. Whitman, J. G. Lim et al., "Hearing symptoms in migrainous infarction," Archives of Neurology, vol. 60, no. 1, pp. 113-116, 2003.

[43] P. A. Bernard and R. J. Stenstrom, "Fluctuating hearing losses in children can be migraine equivalents," International Journal of Pediatric Otorhinolaryngology, vol. 16, no. 2, pp. 141-148, 1988.

[44] G. M. Kalinec, G. Lomberk, R. A. Urrutia, and F. Kalinec, "Resolution of cochlear inflammation: novel target for preventing or ameliorating drug-, noise- and age-related hearing loss," Frontiers in Cellular Neuroscience, vol. 11, no. $192,2017$.

[45] F. Ihler, K. Sharaf, M. Bertlich et al., "Etanercept prevents decrease of cochlear blood flow dose-dependently caused by tumor necrosis factor alpha," Annals of Otology, Rhinology and Laryngology, vol. 122, no. 7, pp. 468-473, 2013.

[46] S. Aminpour, S. P. Tinling, and H. A. Brodie, "Role of tumor necrosis factor- $\alpha$ in sensorineural hearing loss after bacterial meningitis," Otology \& Neurotology, vol. 26, no. 4, pp. 602-609, 2005.

[47] M. Syrakic, S. Pathak, E. Goldofsky et al., "Diagnostic and prognostic utility of measuring tumor necrosis factor in the peripheral circulation of patients with immune-mediated sensorineural hearing loss," Archives of Otolaryngology and Head and Neck Surgery, vol. 138, no. 111, pp. 1052-1058, 2012.

[48] M. Bertlich, F. Ihler, B. G. Weiss, S. Freytag, M. Strupp, and M. Canis, "Cochlear pericytes are capable of reversibly decreasing capillary diameter in vivo after tumor necrosis factor exposure," Otology \& Neurotology, vol. 38, no. 10, pp. e545-e550, 2017.

[49] E. Q. Scherer, J. Yang, M. Canis et al., "Tumor necrosis factor- $\alpha$ enhances microvascular tone and reduces blood flow in the cochlea via enhanced sphingosine-1-phosphate signaling," Stroke, vol. 41, no. 11, pp. 2618-2624, 2010.

[50] A. E. Alewijnse, S. L. M. Peters, and M. C. Michel, "Cardiovascular effects of sphingosine-1-phosphate and other sphingomyelin metabolites," British Journal of Pharmacology, vol. 143, no. 6, pp. 666-684, 2004.

[51] E. Scherer, D. Lidington, E. Oestreicher, W. Arnold, U. Pohl, and S. Bolz, "Sphingosine-1-phosphate modulates spiral modiolar artery tone: a potential role in vascular-based inner 
ear pathologies?," Cardiovascular Research, vol. 70, no. 1, pp. 79-87, 2006.

[52] E. Vila and M. Salaices, "Cytokines and vascular reactivity in resistance arteries," American Journal of Physiology-Heart and Circulatory Physiology, vol. 288, no. 3, pp. H1016H1021, 2005.

[53] S. Morrill and D. Z. Z. He, "Apoptosis in inner ear sensory hair cells," Journal of Otology, vol. 12, no. 4, pp. 151-164, 2017.

[54] T. R. Van de Water, C. T. Dinh, R. Vivero, G. Hoosien, A. A. Eshraghi, and T. J. Balkany, "Mechanisms of hearing loss from trauma and inflammation: otoprotective therapies from the laboratory to the clinic," Acta Oto-Laryngologica, vol. 130 , no. 3, pp. 308-311, 2010.

[55] J. Hiroki, H. Shimokawa, M. Higashi et al., "Inflammatory stimuli upregulate Rho-kinase in human coronary vascular smooth muscle cells," Journal of Molecular and Cellular Cardiology, vol. 37, no. 2, pp. 537-546, 2004.

[56] H. Shimokawa, S. Sunamura, and K. Satoh, "RhoA/rho-kinase in the cardiovascular system," Circulation Research, vol. 118, no. 2, pp. 352-366, 2016.

[57] Y. Mizuno, E. Isotani, J. Huang, H. Ding, J. T. Stull, and K. E. Kamm, "Myosin light chain kinase activation and calcium sensitization in smooth muscle in vivo," American Journal of Physiology-Cell Physiology, vol. 295, no. 2, pp. C358-C364, 2008.

[58] E. Q. Scherer, W. Arnold, and P. Wangemann, "Pharmacological reversal of endothelin-1 mediated constriction of the spiral modiolar artery: a potential new treatment for sudden sensorineural hearing loss," BMC Ear Nose Throat Disorders, vol. 5, no. 10, 2005.

[59] J.-Y. Um, C.-H. Jang, H.-L. Kim et al., "Proinflammatory cytokine IL-1 $\beta$ polymorphisms in sudden sensorineural hearing loss," Immunopharmacology and Immunotoxicology, vol. 35, no. 1, pp. 52-56, 2012.

[60] J.-Y. Um, C.-H. Jang, K.-Y. Kim et al., "Candidate genes of cerebrovascular disease and sudden sensorineural hearing loss," Clinical and Applied Thrombosis/Hemostasis, vol. 16, no. 5, pp. 559-562, 2010.

[61] J.-Y. Um, N.-H. An, and H.-M. Kim, "TNF- $\alpha$ and TNF- $\beta$ gene polymorphisms in cerebral infarction," Journal of Molecular Neuroscience, vol. 21, no. 2, pp. 167-172, 2003.

[62] S. Gupta, R. Zivadinov, D. Ramasamy, and J. L. Ambrus Jr., "Reversible cerebral vasoconstriction syndrome (RCVS) in antiphospholipid antibody syndrome (APLA): the role of centrally acting vasodilators. Case series and review of literature," Clinical Rheumatology, vol. 33, no. 12, pp. 1829$1833,2014$.

[63] I. Uemaetomari, K. Tabuchi, M. Nakamagoe, S. Tanaka, H. Murashita, and A. Hara, "L-type voltage-gated calcium channel is involved in the pathogenesis of acoustic injury in the cochlea," The Tohoku Journal of Experimental Medicine, vol. 218, no. 1, pp. 41-47, 2009.

[64] U.-R. Heinrich, J. Maurer, and W. Mann, "Ultrastructural evidence for protection of the outer hair cells of the inner ear during intense noise exposure by application of the organic calcium channel blocker diltiazem," ORL, vol. 61, no. 6, pp. 321-327, 1999.

[65] H. Shen, B. Zhang, J.-H. Shin et al., "Prophylactic and therapeutic functions of T-type calcium blockers against noise-induced hearing loss," Hearing Research, vol. 226, no. 1-2, pp. 52-60, 2007.

[66] S. B. Minami, D. Yamashita, J. Schacht, and J. M. Miller, "Calcineurin activation contributes to noise-induced hearing loss," Journal of Neuroscience Research, vol. 78, no. 3, pp. 383-392, 2004.

[67] T. Güenther, H. Ising, and H. J. Merker, "Elektrolvt- und Kollagengehalt im Rattenherzen bei chronischem Magnesium-mangel und Stress," Clinical Chemistry Clinical Biochemistry, vol. 16, no. 5, pp. 293-297, 1978.

[68] B. M. Altura and B. T. Altura, "Role of magnesium ions in contractility of blood vessels and skeletals muscles," Magnesium Bulletin, vol. 3, pp. 102-114, 1981.

[69] B. M. Altura, B. T. Altura, A. Gebrewold, H. Ising, and T. Gunther, "Noise-induced hypertension and magnesium in rats: relationship to microcirculation and calcium," Journal of Applied Physiology, vol. 72, no. 1, pp. 194-202, 1992.

[70] M. J. Cevette, J. Vormann, and K. Franz, "Magnesium and hearing," Journal of the American Academy of Audiology, vol. 14, pp. 202-212, 2003.

[71] T. Gunther, H. Ising, and Z. Joachims, "Biochemical mechanisms affecting susceptibility to noise-induced hearing loss," American Journal of Otology, vol. 10, pp. 36-41, 1989.

[72] Z. Joachims, W. Babisch, H. Ising, T. Günther, and M. Handrock, "Dependence of noise-induced hearing loss upon perilymph magnesium concentration," The Journal of the Acoustical Society of America, vol. 74, no. 1, pp. 104-108, 1983.

[73] H. Ising, M. Handrock, T. Günther, R. Fischer, and M. Dombrowski, "Increased noise trauma in Guinea pigs through magnesium deficiency," Archives of Oto-RhinoLaryngology, vol. 236, no. 2, pp. 139-146, 1982.

[74] H. Haupt and F. Scheibe, "Preventive magnesium supplement protects the inner ear against noise-induced impairment of blood flow and oxygenation in the Guinea pig," Magnesium Research, vol. 15, pp. 17-25, 2002.

[75] J. Attias, I. Bresloff, H. Haupt, F. Scheibe, and H. Ising, "Preventing noise induced otoacoustic emission loss by increasing magnesium $\left(\mathrm{Mg}^{2+}\right)$ intake in Guinea-pigs," Journal of Basic Clinical Physiology and Pharmacology, vol. 14, pp. 119-136, 2003.

[76] F. Scheibe, H. Haupt, and H. Ising, "Preventive effect of magnesium supplement on noise-induced hearing loss in the Guinea pig," European Archives of Oto-Rhino-Laryngology, vol. 257, no. 1, pp. 10-16, 2000.

[77] B. I. Nageris, D. Ulanovski, and J. Attias, "Magnesium treatment for sudden hearing loss," Annals of Otology, Rhinology and Laryngology, vol. 113, no. 8, pp. 672-675, 2004.

[78] J. Attias, G. Weisz, S. Almog et al., "Oral magnesium intake reduces permanent hearing loss induced by noise exposure," American Journal of Otolaryngology, vol. 15, no. 1, pp. 26-32, 1994.

[79] Z. Joachims, A. Netzer, H. Ising et al., "Oral magnesium supplementation as prophylaxis for noise-induced hearing loss: results of a double blind field study," Schriftenreihe Verein fuer Wasser und Boden Lufthygiene, vol. 88, pp. 503-516, 1993.

[80] D. Mukherjea, S. Ghosh, P. Bhatta et al., "Early investigational drugs for hearing loss," Expert Opinion on Investigational Drugs, vol. 24, no. 2, pp. 201-217, 2015.

[81] X. Wang, T. Truong, P. B. Billings, J. P. Harris, and E. M. Keithley, "Blockage of immune-mediated inner ear damage by etanercept," Otology \& Neurotology, vol. 24, no. 1, pp. 52-57, 2003.

[82] S. Cohen, A. Shoup, M. H. Weisman, and J. Harris, "Etanercept treatment for autoimmune inner ear disease: results of a pilot placebo-controlled study," Otology \& Neurotology, vol. 26, no. 5, pp. 903-907, 2005. 
[83] X. Han, X. Yin, X. Du, and C. Sun, "Combined intratympanic and systemic use of steroids as a first-line treatment for sudden sensorineural hearing loss," Otology \& Neurotology, vol. 38, no. 4, pp. 487-495, 2017.

[84] N. G. El Sabbagh, M. J. Sewitch, A. Bezdjian, and S. J. Daniel, "Intratympanic dexamethasone in sudden sensorineural hearing loss: a systematic review and meta-analysis," The Laryngoscope, vol. 127, no. 8, pp. 1897-1908, 2017.

[85] H. Li, G. Feng, H. Wang, and Y. Feng, "Intratympanic steroid therapy as a salvage treatment for sudden sensorineural hearing loss after failure of conventional therapy: a metaanalysis of randomized, controlled trials," Clinical Therapeutics, vol. 37, no. 1, pp. 178-187, 2015.

[86] R. A. Crane, M. Camilon, S. Nguyen, and T. A. Meyer, "Steroids for treatment of sudden sensorineural hearing loss: a meta-analysis of randomized controlled trials," The Laryngoscope, vol. 125, no. 1, pp. 209-217, 2015.

[87] Y. Rikitake and J. K. Liao, "Rho GTPases, statins, and nitric oxide," Circulation Research, vol. 97, no. 12, pp. 1232-1235, 2005.

[88] A. Oesterle, U. Laufs, and J. K. Liao, "Pleiotropic effects of statins on the cardiovascular system," Circulation Research, vol. 120, no. 1, pp. 229-243, 2017.

[89] A. Cai, Y. Zhou, and L. Li, "Rho-GTPase and atherosclerosis: pleiotropic effects of statins," Journal of the American Heart Association, vol. 4, no. 7, 2015.

[90] L. Jahani, A. Mehrparvar, M. Esmailidehaj, M. Rezvani, B. Moghbeolohossein, and Z. Razmjooei, "The effect of atorvastatin on preventing noise-induced hearing loss: an experimental study," The International Journal of Occupational and Environmental Medicine, vol. 7, no. 1, pp. 15-21, 2016.

[91] J. S. Park, S. W. Kim, K. Park, Y. H. Choung, I. Jou, and S. M. Park, "Pravastatin attenuates noise-induced cochlear injury in mice," Neuroscience, vol. 208, pp. 123-132, 2012.

[92] C. P. Richter, H. Young, S. V. Richter et al., "Fluvastatin protects cochleae from damage by high-level noise," Scientific Reports, vol. 8, no. 1, p. 3033, 2018.

[93] H. Yuecel, A. Yuecel, H. Arbag, E. Cure, M. A. Eryilmaz, and A. B. Oezer, "Effect of statins on hearing function and subjective tinnitus in hyperlipidemic patients," Romanian Journal of Internal Medicine, 2018, In press.

[94] B. Gopinath, V. M. Flood, E. Teber, C. M. McMahon, and P. Mitchell, "Dietary intake of cholesterol is positively associated and use of cholesterol-lowering medication is negatively associated with prevalent age-related hearing loss," The Journal of Nutrition, vol. 141, no. 7, pp. 1355-1361, 2011.

[95] S.-D. Chung, C.-H. Chen, S.-H. Hung, H.-C. Lin, and L.-H. Wang, "A population-based study on the association between statin use and sudden sensorineural hearing loss," Otolaryngology-Head and Neck Surgery, vol. 152, no. 2, pp. 319-325, 2015.

[96] B. Olzowy, M. Canis, J.-M. Hempel, B. Mazurek, and M. Suckfüll, "Effect of atorvastatin on progression of sensorineural hearing loss and tinnitus in the elderly," Otology \& Neurotology, vol. 28, no. 4, pp. 455-458, 2007.

[97] J. Cai, D. Xu, X. Bai et al., "Curcumin mitigates cerebral vasospasm and early brain injury following subarachnoid hemorrhage via inhibiting cerebral inflammation," Brain and Behavior, vol. 7, no. 9, p. e00790, 2017.

[98] C. Wakade, M. D. King, M. D. Laird, C. H. Alleyne Jr., and K. M. Dhandapani, "Curcumin attenuates vascular inflammation and cerebral vasospasm after subarachnoid hemorrhage in mice," Antioxidants \& Redox Signaling, vol. 11, no. 1, pp. 35-46, 2009.
[99] T. Yamaguchi, M. Yoneyama, Y. Onaka, A. Imaizumi, and K. Ogita, "Preventive effect of curcumin and its highly bioavailable preparation on hearing loss induced by single or repeated exposure to noise: a comparative and mechanistic study," Journal of Pharmacological Sciences, vol. 134, no. 4, pp. 225-233, 2017.

[100] A. Sahebkar, A. F. G. Cicero, L. E. Simental-Mendía, B. B. Aggarwal, and S. C. Gupta, "Curcumin downregulates human tumor necrosis factor- $\alpha$ levels: a systematic review and meta-analysis ofrandomized controlled trials," Pharmacological Research, vol. 107, pp. 234-242, 2016.

[101] S. S. S. Boyanapalli, Y. Huang, Z. Su et al., "Pharmacokinetics and Pharmacodynamics of Curcumin in regulating anti-inflammatory and epigenetic gene expression," Biopharmaceutics \& Drug Disposition, vol. 39, no. 6, pp. 289-297, 2018.

[102] M. Jaumann, J. Dettling, M. Gubelt et al., "cGMP-Prkg1 signaling and Pde5 inhibition shelter cochlear hair cells and hearing function," Nature Medicine, vol. 18, no. 2, pp. 252-259, 2012.

[103] C. A. Peixoto, A. K. S. Nunes, and C. Raposo, "The role of $\mathrm{NO} / \mathrm{cGMP}$ signaling on neuroinflammation: a new therapeutic opportunity," Mechanisms of Neuroinflammation, IntechOpen Limited, London, UK, 2017.

[104] G. McGwin Jr., "Phosphodiesterase type 5 inhibitor use and hearing impairment," Archives of Otolaryngology-Head \& Neck Surgery, vol. 136, no. 5, pp. 488-492, 2010.

[105] F. Giuliano, G. Jackson, F. Montorsi, A. Martin-Morales, and P. Raillard, "Safety of sildenafil citrate: review of 67 doubleblind placebo-controlled trials and the postmarketing safety database," International Journal of Clinical Practice, vol. 64, no. 2, pp. 240-255, 2010.

[106] H.-T. Hsu, J.-Y. Chen, S.-F. Weng, K.-H. Huang, and Y.-S. Lin, "Increased risk of erectile dysfunction in patients with sudden sensorineural hearing loss," Otology \& $\mathrm{Neu}$ rotology, vol. 34, no. 5, pp. 862-867, 2013.

[107] J. J. Keller, Y. K. Chen, and H. C. Lin, "A case-control analysis on the association between erectile dysfunction and sudden sensorineural hearing loss in Taiwan," The Journal of Sexual Medicine, vol. 9, no. 5, pp. 1411-1417, 2012.

[108] M. Dong, B. Yan, and C.-M. Yu, "Current status of rhoassociated kinases (ROCKs) in coronary atherosclerosis and vasospasm," Cardiovascular \& Hematological Agents in Medicinal Chemistry, vol. 7, no. 4, pp. 322-330, 2009.

[109] Q. Zhou, C. Gensch, and J. K. Liao, "Rho-associated coiledcoil-forming kinases (ROCKs): potential targets for the treatment of atherosclerosis and vascular disease," Trends in Pharmacological Sciences, vol. 32, no. 3, pp. 167-173, 2011.

[110] A. Didier, M. T. Droy-Lefaix, C. Aurousseau, and Y. Cazals, "Effects of Gingko biloba extract (EBp 761) on cochlear vasculature in the Guinea pig: morphometric measurements and laser Doppler flowmetry," European Archives of Otorhinolaryngology, vol. 253, pp. 25-30, 1996.

[111] R. Castañeda, S. Natarajan, S. Y. Jeong, B. N. Hong, and T. H. Kang, "Traditional oriental medicine for sensorineural hearing loss: can ethnopharmacology contribute to potential drug discovery?," Journal of Ethnopharmacology, vol. 231, pp. 409-428, 2019.

[112] E. Ponzetto, M. Vinetti, C. Grandin et al., "Partly reversible central auditory dysfunction induced by cerebral vasospasm after subarachnoid hemorrhage," Journal of Neurosurgery, vol. 119, no. 5, pp. 1125-1128, 2013.

[113] K. Hyun-Ah and L. Hyung Lee, "Recent advances in understanding audiovestibular loss of a vascular cause," Journal of Stroke, vol. 19, no. 1, pp. 61-66, 2017. 


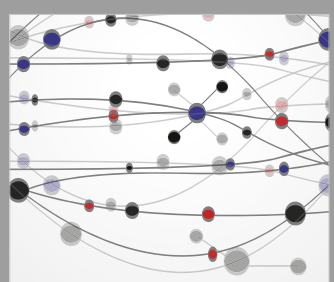

The Scientific World Journal
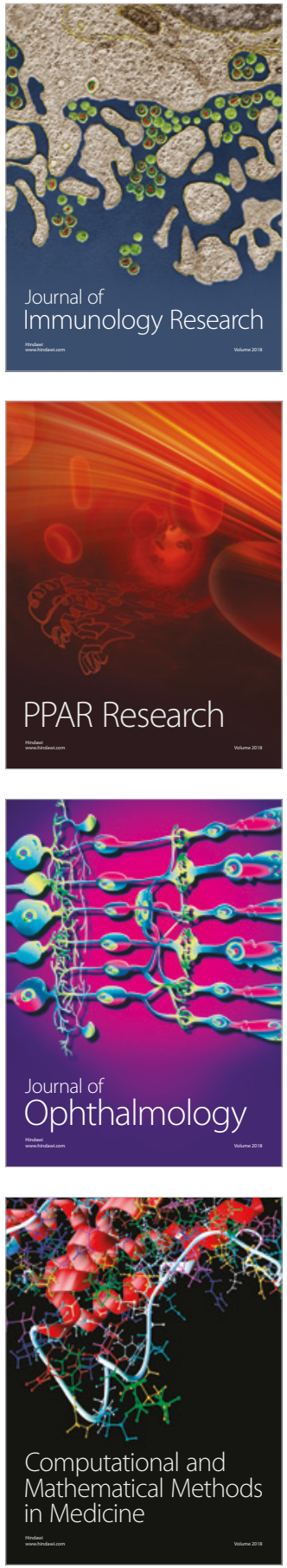

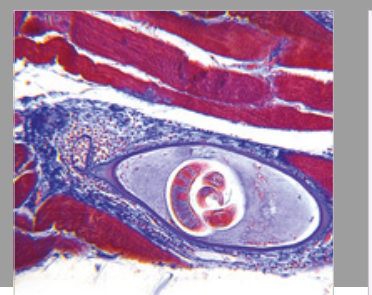

Gastroenterology Research and Practice

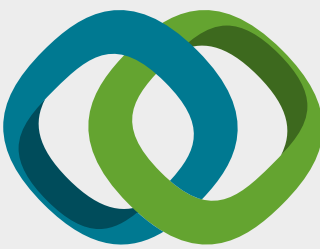

\section{Hindawi}

Submit your manuscripts at

www.hindawi.com
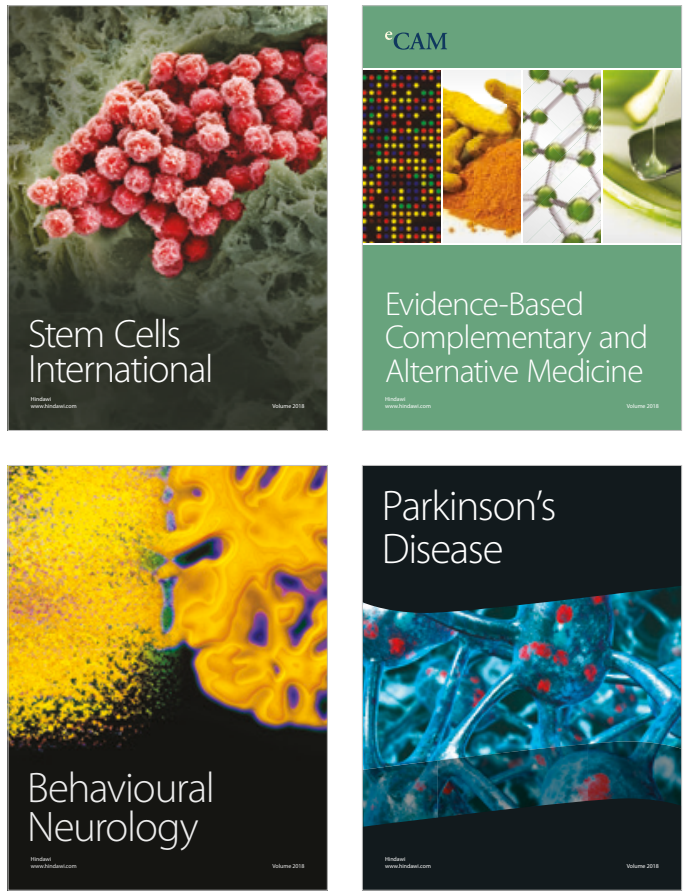

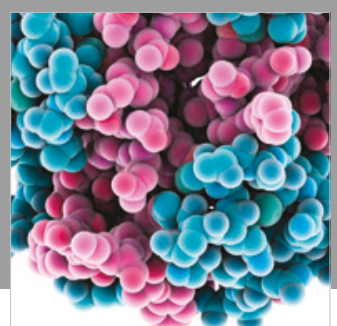

ournal of

Diabetes Research

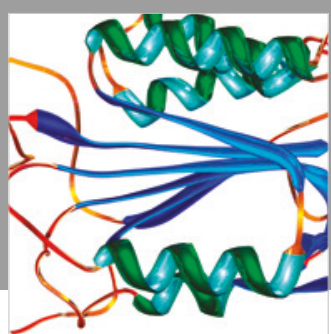

Disease Markers
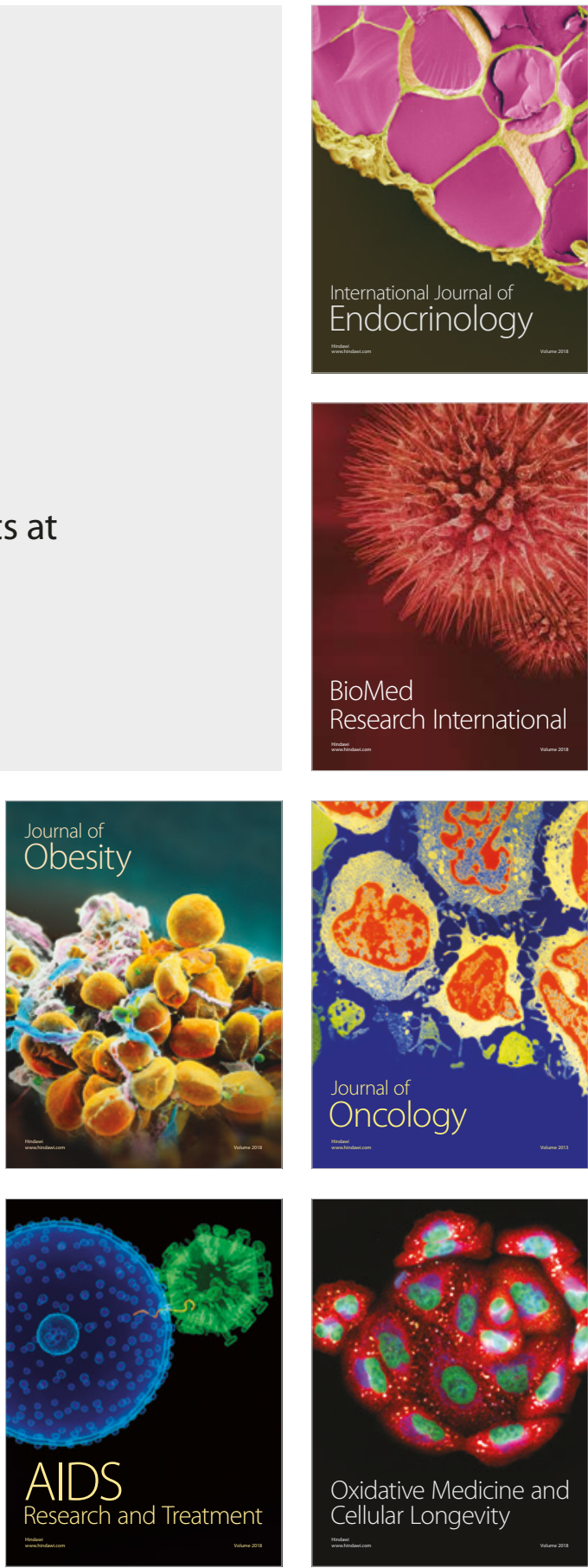